\title{
The time for emergency department opioid agonist therapy is now: "A BC perspective"
}

\author{
Andrew Kestler, MD, MBA, MScPH, DTMH*+‡; Jason Wale, MD*§ף; Melissa Allan, MD ${ }^{* * * \dagger \dagger}$
}

Although we have national evidence-based guidelines to address Canada's opioid epidemic, ${ }^{1}$ the number of annual opioid overdose deaths continues to rise. From January 2016 to September 2018, 10,337 Canadians died of opioid-related overdoses, ${ }^{2}$ with British Columbia (BC) a regrettable national leader. Patients and providers need better access to the proven intervention of opioid agonist therapy, which includes methadone and buprenorphine/naloxone (often referred to as Suboxone, or $B N X)$.

Opioid use disorder kills more effectively than diseases that have historically received emergency department (ED) attention and resources. In an American cohort prior to widespread fentanyl use, $4.7 \%$ of individuals treated for opioid overdoses were dead at 1 year. ${ }^{3}$ In Vancouver, ED patients treated for overdose in 2017 had an 8\% 1-year mortality (Jiang and Purssell, personal communication, April 2019).

Opioid agonist therapy cuts overdose and all-cause mortality in half in people with opioid use disorder, ${ }^{3,4}$ but fewer than $30 \%$ of people with diagnosed opioid use disorder receive 1 month of opioid agonist therapy, whether in Massachusetts ${ }^{3}$ or in BC (Nosyk, personal communication, April 2019). Across the United States and Canada, rates of people with opioid use disorder receiving adequate opioid agonist therapy are likely much lower if one considers 1) the heterogeneity of addiction treatment resources, 2) the burden of undiagnosed opioid use disorder, and 3) the longer opioid agonist therapy duration thought necessary to maximize survival benefit. If initiated in the ED, buprenorphine/ naloxone (the first line opioid agonist therapy choice in
Canada $^{1}$ ) doubles rates of retention in addiction care compared with a passive referral alone. ${ }^{5}$

Every ED encounter constitutes an opportunity to start opioid agonist therapy before the next fatal overdose. In BC, over $50 \%$ of people dying of overdose visited an ED in the year before death. ${ }^{6}$ Sadly, some ED opioid use disorder patients feel stigmatized ${ }^{7}$ and leave before being seen. Others pass through EDs with unrecognized opioid use disorder, often with triage complaints that do not appear opioid-related. Still others may be dismissed as "drug seekers" when they are actually providing clues to underlying opioid use disorder. Nevertheless, a challenging ED visit does not preclude later opioid agonist therapy success. Many opioid use disorder survivors become the peer support workers so crucial to helping others enter recovery.

In this issue of $C F E M, \mathrm{Hu}$ and colleagues ${ }^{8}$ give cause for optimism: They demonstrate that Canadian community hospital EDs can initiate buprenorphine/naloxone and, via community linkages, successfully maintain patients on buprenorphine/naloxone for 6 months, incidentally lowering the number of repeat ED visits during follow-up.

A cautionary note: $\mathrm{Hu}$ and colleagues' protocol $^{8}$ initiates buprenorphine/naloxone at a Clinical Opioid Withdrawal Scale (COWS) score greater than 5. Participants, in fact, reached an average COWS of 11 before starting buprenorphine/naloxone (Hu, personal communication, April 2019). Guidelines in $\mathrm{BC}^{9}$ call for a minimum COWS score of 12 before beginning buprenorphine/ naloxone. Starting with lower scores increases the risk of precipitated withdrawal.

From the *Faculty of Medicine, University of British Columbia, Vancouver, BC; †St. Paul’s Hospital, Vancouver, BC; $¥$ Centre for Health Evaluation \& Outcome Sciences, Vancouver, BC; §Royal Jubilee and Victoria General Hospitals, Victoria, BC; TVancouver Island Health Authority, Vancouver, BC; **Burnaby Hospital, Burnaby, BC; and the ††Fraser Health Authority, Surrey, BC.

Correspondence to: Dr. Andrew Kestler, Department of Emergency Medicine, University of British Columbia and St. Paul's Hospital, 1081 Burrard Street, Vancouver, BC V6Z 1Y6; Email: andrew.kestler@ubc.ca

(C) Canadian Association of Emergency Physicians 
Many barriers remain to ED opioid agonist therapy success. ED staff have biases affecting their care of patients with substance use disorders. ${ }^{10}$ ED physicians in Saskatchewan and Manitoba still need special approval to initiate buprenorphine/naloxone for their patients, ${ }^{1}$ and buprenorphine/naloxone is not uniformly covered for all patients in all provinces. ${ }^{1}$ Many areas lack the essential community follow-up piece of ED opioid agonist therapy.

The Canadian Research Initiative on Substance Misuse (CRISM, https://crism.ca/) has launched a substantive effort to better understand ED opioid agonist therapy from both the patient and provider perspectives. CRISM plans to use its findings to develop and disseminate an ED opioid agonist therapy toolkit specific to reducing implementation barriers in the Canadian context. Of course, no single approach or tool will work for all settings. In BC alone, different EDs have devised different opioid agonist therapy programs to suit their patient population and their resources.

In Vancouver, in 2017, St. Paul's Hospital ED began offering buprenorphine/naloxone to its patients. However, few patients, many of whom inject drugs daily, were in sufficient opioid withdrawal to initiate buprenorphine/naloxone. In response, the ED launched a lowbarrier buprenorphine/naloxone to-go program, where patients receive a starter pack with instructions regarding self-administration and follow-up. So far, $25 \%$ patients are retained on opioid agonist therapy on follow-up. The new program is linked to a rapid access addiction clinic and a community outreach team, which successfully finds $90 \%$ of patient post ED-discharge and links approximately 50\% to ongoing care (Bath, personal communication, April 2019).

In Burnaby, proportionally fewer people with opioid use disorder inject drugs than in Vancouver, and more present to ED in moderate opioid withdrawal. With minimal resources, the ED began to offer buprenorphine/naloxone in 2017 and found community physicians to see patients in follow-up. The ED program has since garnered Fraser Health Authority support for several new components: universal screening for overdose risk; funded community opioid agonist therapy clinics; outreach teams to follow up all patients who overdose or start opioid agonist therapy; staff education on trauma informed care to reduce opioid-related stigma; and provider resiliency training to boost staff morale and compassion. The Authority is expanding the program to its 12 hospitals, in a regional model not unlike what $\mathrm{Hu}$ describes at Lakeridge Health in Ontario. ${ }^{8}$

In Victoria, peer support workers play a large role in ED opioid agonist therapy. The Victoria Umbrella Society is a non-profit organization of peers in recovery from addiction. The peers contact patients started on ED opioid agonist therapy or identified in the ED as opioid agonist therapy candidates in order to optimize transition to outpatient care. Since March 2017, the peers have helped connect 150 patients to ongoing care with more than 30 remaining stable on opioid agonist therapy for over 3 months. The ED observational unit accepts patients in early withdrawal for up to 23 hours, allowing patients to reach sufficient withdrawal to start buprenorphine/naloxone treatment in the ED before peerassisted follow-up in the community.

Guidelines, growing evidence, and mortality statistics are telling us we should be offering more take-home naloxone, ED opioid agonist therapy, and communitybased addiction care to our patients with opioid use disorder. Yet many of us still work in systems that make treating opioid use disorder needlessly more difficult than complex conditions like septic shock and decompensated heart failure. It is then our collective responsibility to demand the institutional and regulatory support we all need to offer opioid agonist therapy to our patients now, before it is too late.

Keywords: Buprenorphine, emergency department, opioid agonist therapy, opioid use disorder

Competing interests: None declared.

\section{REFERENCES}

1. Canadian Research Initiative in Substance Misuse. CRISM national guideline for the clinical management of opioid use disorder. CRISM; 2018. Available at: https://crism.ca/ projects/opioid-guideline/ (accessed April 30, 2019).

2. Special Advisory Committee on the Epidemic of Opioid Overdoses. National report: apparent opioid-related deaths in Canada. Ottawa: Public Health Agency of Canada; 2019. Available at: https://infobase.phac-aspc.gc.ca/datalab/ national-surveillance-opioid-harms-mortality.html (accessed April 30, 2019).

3. Larochelle MR, Bernson D, Land T, et al. Medication for opioid use disorder after nonfatal opioid overdose and association with mortality. Ann Intern Med 2018;169:137-145.

4. Sordo L, Barrio G, Bravo MJ, et al. Mortality risk during and after opioid substitution treatment: systematic review and meta-analysis of cohort studies. BM7 2017;357:j1550. 
5. D'Onofrio G, O'Connor PG, Pantalon $M V$, et al. Emergency department-initiated buprenorphine/naloxone treatment for opioid dependence. FAMA 2015;313:1636.

6. Otterstatter MC, Crabtree A, Dobrer S, et al. Patterns of health care utilization among people who overdosed from illegal drugs: a descriptive analysis using the BC Provincial Overdose Cohort. Health Promot Chronic Dis Prev Can 2018;38:328-38.

7. Kahan D, Poremski D, Wise-Harris D, et al. Perceived case management needs and service preferences of frequent emergency department users: lessons learned in a large urban centre. PLoS One 2016;11:e0168782.
8. Hu T, Snider-Adler M, Nijmeh L, Pyle A. Buprenorphine/ naloxone induction in a Canadian emergency department with rapid access to community-based addiction providers. C7EM 2019; epub, doi:10.1017/cem.2019.24.

9. British Columbia Centre on Substance Use, British Columbia Ministry of Health. A guideline for the clinical management of opioid use disorder; 2017. Available at: http://www.bccsu.ca/care-guidance-publications/ (accessed April 30, 2019).

10. Mendiola CK, Galetto G, Fingerhood M. An exploration of emergency physicians' attitudes toward patients with substance use disorder. 7 Addict Med 2018;12:132-5. 